\title{
Individual and geographic variations in the propensity to travel by active modes in Vitoria-Gasteiz, Spain
}

\author{
Belén Martín ${ }^{\mathrm{a}, \mathrm{b}, *}$, Antonio Páez ${ }^{\mathrm{c}}$ \\ ${ }^{a}$ Department of Forest and Environmental Engineering and Management, MONTES (School of Forest Engineering and Natural Environment), Universidad Politécnica de \\ Madrid, 28040 Madrid, Spain \\ ${ }^{\mathrm{b}}$ Transport Research Centre (TRANSyT-UPM), Universidad Politécnica de Madrid, 28040 Madrid, Spain \\ ${ }^{\mathrm{c}}$ McMaster Institute for Transportation and Logistics, McMaster University. School of Geography and Earth Sciences, 1280 Main St W, Hamilton, ON L8S 4K1, Canada
}

A R T I C L E IN F O

\section{Keywords:}

Active transport mode

Spatially-varying coefficients

Geographic profiles

Land use mix

Age

Gender

\begin{abstract}
A B S T R A C T
The transportation impacts of an increasingly urban population have led planners and policy makers to consider ways to encourage the use of active travel in urban areas. Active travel is attractive from a planning perspective because its environmental impact is considerably lower than the alternatives, and in addition is known to have a number of population health and social benefits. Not surprisingly, there has been a growth in interest in the factors associated with the use of active modes of transportation. The objective of this study is to investigate the individual (demographic and socio-economic) and contextual (geography and land use type) factors that correlate with the propensity to travel by active modes in Vitoria-Gasteiz. This is a medium sized city in the north of Spain, and a case study that has hitherto not been reported. Data obtained from a household travel survey (HTS) conducted in 2014 allow us to estimate a multinomial logit model of the propensity to travel by different modes of transportation. Furthermore, since the data are geocoded, we can estimate a model with spatially-varying coefficients to assess the geographical variations in the probability to use active modes. The results of the study provide valuable information concerning differences in active travel by gender and age, including significant and quite substantial variations by location. In addition, the effect of different urban fabrics indicates the relevance of density and mix of uses for active travel. In this way, the present research helps to increase our global knowledge-base regarding active travel in a medium-sized city.
\end{abstract}

\section{Introduction}

The size of the world's urban population continues to increase. According to the United Nations (2014) 54\% of the world population is now urban, and the forecast is that this percentage will reach $60 \%$ by 2050. This percentage is even higher for countries with developed economies. The rate of urbanization presents challenges to urban planners, who are tasked with helping to manage this growth in a sustainable way - with transport planning playing a crucial part to achieve this objective. In recent years, this challenge has also presented renewed opportunities for implementing transport policies to support and promote active transport modes, namely walking and cycling.

The benefits of active travel for urban sustainability and population health are well documented in the literature. Active mobility, for instance, reduces motor vehicle use along with its negative impacts in terms of air pollution, needs for space, and traffic congestion
(Christiansen et al., 2016; Kim and Macdonald, 2016). Active modes do not contribute to greenhouse emissions, noise, or contamination, and do not rely extensively on non-renewable resources. As such, active forms of travel are the only truly sustainable transportation modes. Furthermore, active modes are accessible to the majority of the population, regardless of their income level (Pucher and Buehler, 2008; International Transport Forum, 2012), and walking in particular is recommended as a suitable form of transportation for people of all ages (US Department of Health and Human Services and others, 2008). Walking, in fact, is a fundamental mode in inter-modal travel chains, since every trip begins and ends by walking, and it is also an important source of accessibility on its own right (Litman, 2003). Walking and cycling for transportation can also contribute to daily physical activity (Moniruzzaman et al., 2014; De Hartog et al., 2010), and therefore can also yield important health benefits by helping to reduce the risk of diseases such as cardiovascular disease, diabetes, some cancers, and

\footnotetext{
* Corresponding author at: Department of Forest and Environmental Engineering and Management, MONTES (School of Forest Engineering and Natural Environment), Universidad Politécnica de Madrid, 28040 Madrid, Spain.

E-mail addresses: belen.martin@upm.es (B. Martín), paezha@mcmaster.ca (A. Páez).
} 
depression (World Health Organization, 2017).

In addition to the individual and environmental advantages afforded by walking, this mode is also positive for well-being and social cohesion, since it increases the opportunities for social interaction and permits engagement with local opportunities (Gatrell, 2013; Zhu et al., 2014; Grannis, 2009). As a consequence, walkable environments are associated with sense of community (Whalen et al., 2012). Whereas walking has the potential to substitute some motorized trips (e.g., Morency et al., 2007) the reality is that it has a more limited range. Cycling, in contrast, is a feasible alternative to larger number of motorized trips, and has the advantage of having much lower direct and indirect costs in terms of public infrastructure and space use relative to other road-based modes (Pucher and Buehler, 2008; Lois, Moriano, and Rondinella, 2015).

Given the many potential benefits of active travel for urban environments and population health, it is not surprising that understanding the factors that encourage or deter active travel have attracted considerable attention in recent years. For example, age and gender are variables frequently used to explain travel behavior in different contexts. Previous studies have found that mobility tends to be lower among younger and older people compared to other groups (Paez et al., 2007; Mercado and Paez, 2009; Schmocker et al., 2008), and that seniors travel less, use transit and walk more, and use the car less (Bocker et al., 2017; Tilley and Houston, 2016; Kim and Ulfarsson, 2004). Gender differences are also observed in previous studies, which report that women are more reliant on slower modes, such as walking and transit (Best and Lanzendorf, 2005).

Socioeconomic status has been shown to be influential in the use of different modes of transport. For instance, Simons et al. (2017) report differences in mode use in 3 groups of young adults with different occupations, taking into account their gender and living environment. Among other findings, they report that secondary-school students were more likely to cycle, young adult students were more likely to walk and use public transport, and working young adults were more likely to use a car. They also found that urban young adults were more likely to walk compared to those living in rural areas. Student and working young adults in urban areas were more likely to cycle than those from rural areas. Goodman, Anna (2013), investigated the implications of socioeconomic patterns in different modes in England and Wales, among other findings, the study confirmed that active mobility increases more rapidly in socio-economically advantaged groups, which may rise to inequalities with respect to health results.

The use of different modes is also influenced by urban configuration, structure and geographical context. Concentration of urban land uses in monocentric cities encourages cycling, walking and the use of public transport, while dispersion of urban land uses encourages driving (Schwanen et al., 2001). Investigations in Nordic cities have reported that those living in the outer or less dense parts of the city travel by motorized means of transportation, compared to those living in central parts (Naess, 2012). Recently (Steinmetz-Wood and Kestens, 2015) found interactions between socioeconomic status and the relationship between active travel and built environment measures (density of business and service destinations, connectivity and land-use mix). Potoglou and Arslangulova (2017) examined the factors that influence active travel to school. They demonstrated that environmental factors - distance to school and level of urbanization - together with other personal and household characteristics, were associated with active travel of children in wales. Living within one mile from school and residing in an urban area were positively associated with active travel.

Previous studies that examined the factors that correlate with active travel have a number of limitations. As reported by (Mackenbach et al., 2016), studies on active travel and urban configuration frequently focus on the characteristics of residential areas (Kerr et al., 2013; Saelens et al., 2003). Other areas, such as industrial fabrics, are also important in travel behavior, especially for commuter trips. The characteristics at the start of non-home-based trips are relevant. The influence of urban form in travel behavior at the city scale has often found to be significant. Despite this, although it is widely accepted in the literature that urban structure is relevant in travel behavior in large metropolitan areas (Ewing and Cervero, 2010; Naess et al., 2017; Stevens, 2017), smaller cities are not well represented in the literature (Naess and Jensen, 2004; Wolday, 2018). Research on active travel in small and medium-sized cities is of interest. The number of motorized trips that could potentially be transferred to active modes in these cities could be higher, since trips in general are likely to be shorter - and, as a consequence, in the range of distance that can be walked or cycled (Morency et al., 2014; Delso et al., 2018). Furthermore, the abovementioned works on active modes that use socio-demographic and urban configuration variables to better understand the propensity to travel by active modes do not consider their variation by location. The analysis of variability by location make it make possible to obtain maps of geographical trends that help to understand travel behavior in the city (Roorda et al., 2010; Morency et al., 2011; Moniruzzaman et al., 2013).

The objective of this paper is to investigate the factors that influence mode use in Vitoria-Gasteiz. This is a medium-sized city with a population of approximately 250,000 people in the north of Spain. Geocoded data from a mobility survey conducted in 2014 provides micro-data on, among other items, mode of travel. Estimation of a multinomial model allows us to estimate the probability of traveling by different modes of transportation, with a particular focus on active travel. The propensity of travel by mode in this analysis is a function of individual socioeconomic and demographic attributes. In addition, we examine the role of urban configuration and geographical context. The results of the study provide valuable information concerning active travel by gender and across the age span. In addition, the effect of different urban fabrics indicates the relevance of density and mix of uses for active travel. In this way, the present research helps to increase our global knowledgebase regarding active travel in a medium-sized city.

The paper is structured as follows. The current Section 1 includes the introduction and objectives, Section 2 describes the Vitoria-Gasteiz case study, the data used, and the modeling approach adopted. Section 3 details the obtained results and their discussion. Finally, Section 4 provides concluding remarks and pointers to future research topics.

\section{Data and methods}

\subsection{Context}

The present research is based on the city of Vitoria-Gasteiz, a medium-sized population center with a population of 247,820 inhabitants (Ayuntamiento de Vitoria-Gasteiz, 2016). The city is located in Alava province, in northern Spain, and is characterized by gentle orography and moderately cold continental climate. Local authorities have striven to implement sustainable transportation policies for over a decade, as articulated in the Mobility and Public Space Plan and in the Cycling Mobility Transport Plan (Muñoz and Rondinella, 2017). Among the policies considered in these plans and implemented by the city, there are traffic regulations that consider active transport modes together with cars and public transportation, in addition to measures to increase pedestrian spaces in the city, the promotion of cycling, progressive use of parking fees, and camera-controlled access to the city center. The result is that walking and cycling in Vitoria-Gasteiz have grown substantially over the past few years, with the shares of cycling and walking trips now being among the highest of European mediumsized cities (Muñoz, 2016).

Vitoria-Gasteiz is the capital of the Basque Country Region (Spain) and has evolved from an intensive industrial economic model towards a mixed economy with greater presence of the service sector. As seen in Fig. 1, there is a variety of land uses in the city, with three industrial areas concentrated in the west, north east and south of the city, and a 


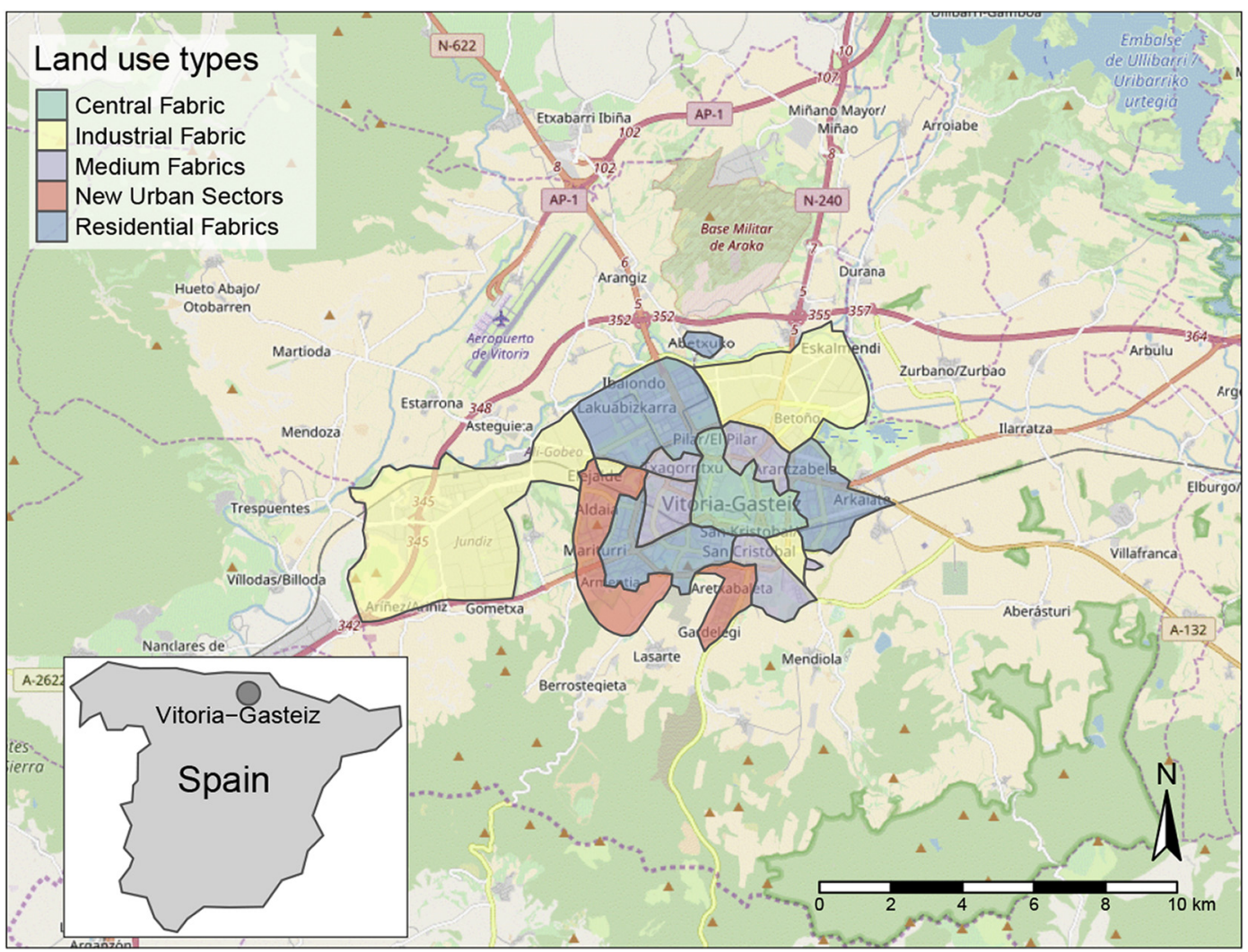

Fig. 1. Typology of urban land uses in Vitoria-Gasteiz.

(Source: Rondinella, 2015).

land-use mix of commercial and residential zones, parks and open spaces in the rest of the city (Ayuntamiento de Vitoria-Gasteiz, 2011). The classification of urban fabric (i.e., land use types) is shown in Fig. 1. The source for the urban fabric types is the work of Rondinella (2015), who in turn based it on the typology commissioned by the City Council and developed by the Agencia de Ecolog Ãa Urbana de Barcelona (Agencia de Ecología Urbana de Barcelona, 2010). As such, it is the tool used by the city for developing policy regarding land use and transportation.

The urban fabrics were edited for geospatial analysis using ArcMap 10.3.1 (ESRI, 2015) and their characteristics are shown in Table 1, including their typical population density and intensity of the built surface as measured by the floor-area ratio (see Rondinella, 2015). Examples of typical configurations of the different land use types are shown in Fig. 2.

Like many European cities, one of the main characteristics of the built environment in Vitoria-Gasteiz is its compactness, and there is a clear separation between urban uses and rural uses in the countryside. The spatial configuration of the city is such that around $32 \%$ of the population lives within $1000 \mathrm{~m}$ from the core of the city center, $74 \%$ within $2000 \mathrm{~m}$, and $96 \%$ within $3000 \mathrm{~m}$ (Rondinella, 2015).

\subsection{Description of the data}

The source of data for this research is the Vitoria-Gasteiz Household Travel Survey (HTS), a one-day travel survey conducted on a weekday during the spring and autumn of 2014 in the region. The survey was telephone-based and asked participants about their trips in the preceding day- with interviews conducted from Tuesdays to Fridays. To be eligible to participate in the survey, respondents had to be at least 9 years old. The random sampling design of the survey was stratified by transport zone ( 40 zones), age group ( 5 groups), and gender. In addition, sampling weights were calculated to reach stratum quotas, and in this way, the weighted sample is representative of the population. The data collection exercise is documented in full in Muñoz (2016).

The total sample for the survey is 4192 individuals who, with the sample weights, represent a population of 218,515 individuals 9 years or older in Vitoria-Gasteiz. The total number of trips captured by the survey (population weighted) is 911,326 , which represents an average of 4.3 trips per person per day. The rate of immobility, on the other hand, is $3.3 \%$, meaning that approximately 7211 individuals did not make any trips in the preceding day.

The preponderance of travel by different modes in the region under study is summarized in Table 2 . The modeling to follow is based on the

Table 1

Land use typology.

Source: Rondinella (2015).

\begin{tabular}{|c|c|c|c|}
\hline Land use type & Density & Floor-Area Ratio & Land use mix \\
\hline Central & $>150$ households/ha & $>1.5$ & Mixed \\
\hline Medium Type & 60 to 150 households/ha & 1 to 1.5 & Mainly residential, some commercial \\
\hline Residential & $<60$ households/ha & $<1$ & Almost exclusively residential, some basic commercial \\
\hline New Urban Sector & 48 to 70 households/ha & Variable & Variable, from residential to highly mixed \\
\hline Industrial & $\mathrm{N} / \mathrm{A}$ & N/A & Industrial \\
\hline
\end{tabular}


Central

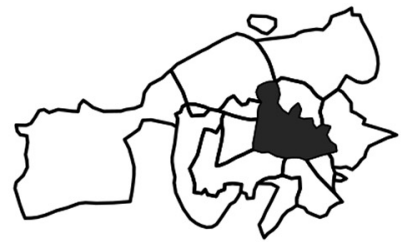

\section{Medium Fabrics}

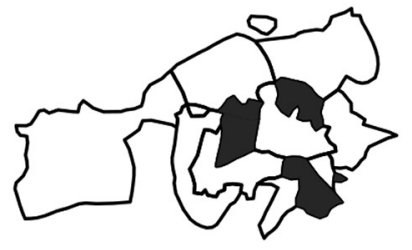

\section{Residential}

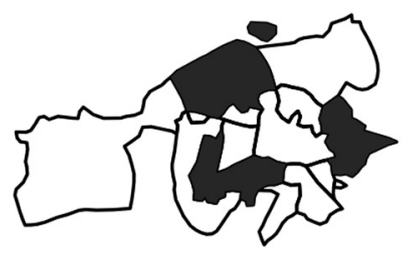

\section{New Urban Sectors}

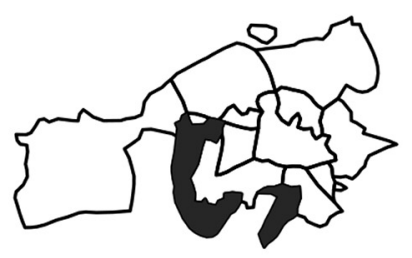

\section{Industrial}

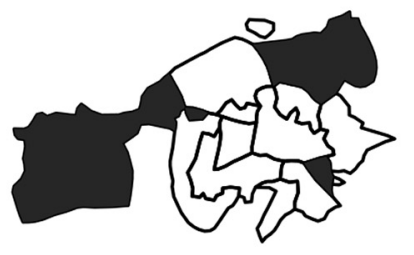

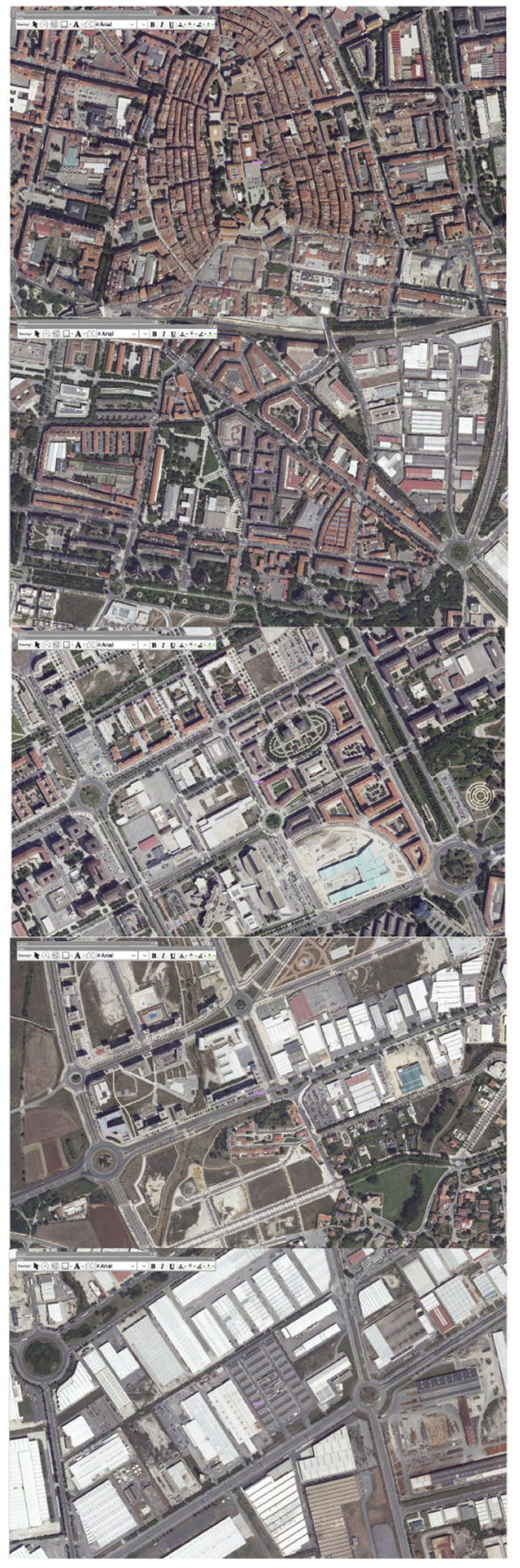

Fig. 2. Examples of typical land use configurations.
Table 2

Mode distribution in the HTS survey sample.

\begin{tabular}{lll}
\hline Mode & Trips (sample) & Weighted trips (population) \\
\hline Walking & 9925 & 494,987 \\
Bicycle & 2165 & 110,635 \\
Car-Driver & 2972 & 173,039 \\
Car-Passenger & 531 & 28,843 \\
Bus & 820 & 40,600 \\
\hline
\end{tabular}

five most frequent modes, specifically two active modes (walking and cycling) and three motorized modes (car as driver, car as passenger, and bus). Together, these five modes account for $95 \%$ of all trips. Modes not considered for the analysis due to their small frequency are taxi, motorbike, and other individual (e.g., van) or collective (institutional bus) modes.

\subsection{Variables}

The dependent variable in the analysis is the mode of transport registered for each trip in the HTS. As noted above, two active modes and three motorized modes are considered. The independent variables, on the other hand, describe socio-economic and demographic attributes of the individual, concretely age, gender, and occupation, as well as the urban land use type at the point of origin of the trip. The independent variables were selected based on a scan of the literature (as outlined in the introductory section).

The sources for the data are as follows. Age, gender, occupation, and population weights were extracted directly from the HTS survey. All trip origins were geocoded based on the addresses reported in the HTS 
survey. Geocoding was conducted using a Python client for Google Maps Services (GitHub Contributors, 2018). The coordinates of the trip origins enter the analysis in two ways: first, they help us to situate the trip origin within the context of a specific urban fabric, and also are used as independent variables to obtain spatially-varying coefficients.

\subsection{Modeling approach}

The canonical modeling approach for a variable such as mode of travel is the multinomial logit model. When individual-level attributes are available, as in the present case, the model is of the probability of a certain outcome corresponding to one of $K$ possible states.

The multinomial logit model can be obtained by defining a set of binary logistic regressions, in the form of the log of the ratio of odds with respect to a reference outcome (say $K$ ), as follows:

$$
\begin{array}{r}
\ln \left(\frac{P\left(Y_{i}=1\right)}{P\left(Y_{i}=K\right)}\right)=X \beta_{1}=\sum_{j=0}^{J} \beta_{1, j} x_{j} \\
\ln \left(\frac{P\left(Y_{i}=2\right)}{P\left(Y_{i}=K\right)}\right)=X \beta_{2}=\sum_{j=0}^{J} \beta_{2, j} x_{j} \\
\ln \left(\frac{P\left(Y_{i}=K-1\right)}{P\left(Y_{i}=K\right)}\right)=X \beta_{K-1}=\sum_{j=0}^{J} \beta_{K-1, j} x_{j}
\end{array}
$$

Each equation above is simply a binary logistic regression with respect to outcome $K$. Importantly, each regression $k$ has its own set of coefficients $\beta_{k}$. Furthermore, since the sum of all probabilities must be 1 , it follows that:

$P\left(Y_{i}=K\right)=1-\sum_{k=1}^{K-1} P\left(Y_{i}=k\right)$

And since we can express each probability in terms of $P\left(Y_{i}=K\right)$, then:

$P\left(Y_{i}=K\right)=1-\sum_{k=1}^{K-1} P\left(Y_{i}=k\right) e^{X \beta_{k}}$,

which, after some manipulation becomes:

$P\left(Y_{i}=k\right)=\frac{1}{1+\sum_{k=1}^{K-1} e^{X \beta_{k}}}$

Given $P\left(Y_{i}=k\right)$, the probability for outcome $k \neq K$ is obtained as follows:

$P\left(Y_{i}=k\right)=P\left(Y_{i}=K\right) e^{X \beta_{k}}$

The availability of detailed geocoding in the form of the coordinates allows us to obtain spatially-varying coefficients by means of the spatial expansion method of Casetti (1972). The expansion method is a simple and intuitive strategy to generate models that allow an analyst to infer systematic spatial trends, and to identify person-location variations.

Implementation of the expansion method is as follows: first, an initial model is posited that contains substantive knowledge about the process of interest. Secondly, some or all of the coefficients in the initial model are expanded as a function of contextual variables. In the case of spatial expansions, the contextual variables are often the coordinates of the observations, and the function a polynomial of a certain order (e.g. linear, quadratic, cubic). Finally, the expanded coefficients are introduced in the terminal model.

Consider the $k$-th equation in the ensemble of regressions that underlie the multinomial logit model:

$\ln \left(\frac{P\left(Y_{i}=k\right)}{P\left(Y_{i}=K\right)}\right)=X \beta_{k}=\sum_{j=0}^{J} \beta_{k, j} x_{j}$

Based on this equation, take an arbitrary coefficient, say $\beta_{k, j}$, for expansion as a function of the coordinates $\left(u_{j}, v_{j}\right)$ at location $j$ :

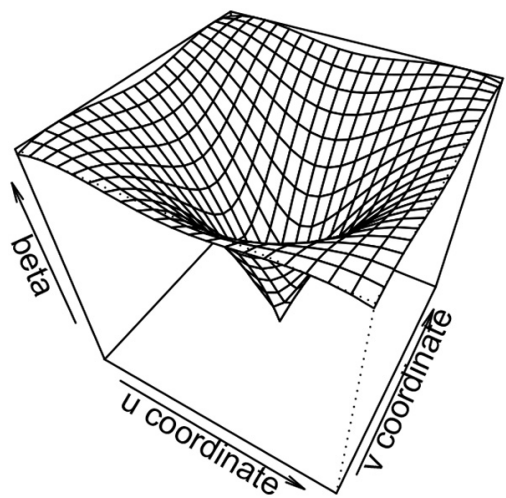

Fig. 3. Example of a spatially-varying coefficient using a quadratic polynomial and distance to CBD.

$\beta_{k, j}=\beta_{k, j}\left(u_{j}, v_{j}\right)$

The spatial expansion that we consider below is a combination of a quadratic trend surface and distance to the central business district (CBD) of the city, as follows:

$\beta_{k, j}=\beta_{k, j}^{0}+\beta_{k, j}^{1} u_{j}^{2}+\beta_{k, j}^{2} u_{j}+\beta_{k, j}^{3} u_{j} v_{j}+\beta_{k, j}^{4} v_{j}+\beta_{k, j}^{5} v_{j}^{2}+\beta_{k, j}^{6} d_{j, C B D}$

By spatially expanding some (or all) coefficients in a model, it is possible to obtain response surfaces that capture geographical trends in the outcome. For instance, Fig. 2 shows an example of the kind of surface that results from using the expansion above. This kind of surface has been employed to represent geographical variations in travel behavior research by Roorda et al. (2010) for trip generation analysis, Morency et al. (2011) for trip length analysis, and Moniruzzaman et al. (2013) for joint mode use and trip length analysis (Fig. 3).

Since the coefficients are spatially-varying, it follows that for a given value of the substantive attribute (say Age), the probability of using a mode is not necessarily a constant, but rather could vary geographically. The usual diagnostics of the coefficients (i.e., $t$-scores or $p$ values) are used to decide whether the components of the spatial expansion are significant or not.

\section{Results and discussion}

The result of estimating the regression model for travel by mode in Vitoria Gasteiz are presented in Table 3. As seen there, the variables Age (and Age2, the square of age), Female, and those related to occupational status were expanded using the coordinates of the trip origin and the distance to the city center using a 2nd order polynomial function. Additionally, indicator variables to describes the type of land use were included in the model. With only two exceptions (the expansion of Age2 with distance to CBD for bicycle and bus), all variables achieve significance at conventional levels of confidence or better.

The goodness of fit of the model is assessed using McFadden's pseudo $-\rho^{2}$, which relates the likelihood value of the full model to the likelihood value of the null model (i.e., a model with no predictors). The model reported a pseudo $-R^{2}$ of 0.16 .

With respect to mode of travel and land use, it is worthwhile noting that the mode of reference is walking, and the reference land use is the central fabric. With this in mind, it is possible to see that the probability of traveling by modes other than walking is higher in every single land type beyond the central fabric. In particular, we see that trips that begin in any of the Industrial fabrics have a significantly higher probability of being by car, especially as passenger. It is also interesting to see that New Urban fabrics are very similar to Rural in terms of the way they associate with travel by car, even if the probability of traveling by bicycle is higher in Rural regions. As seen in Table 1, New Urban fabrics tend to have lower density and are more heterogeneous than the Central, Residential, and Medium Type fabrics. Instead, Medium and 
Table 3

Multinomial logit model with spatially expanded coefficients.

\begin{tabular}{|c|c|c|c|c|c|c|c|c|}
\hline \multirow[b]{2}{*}{ Variable } & \multicolumn{2}{|l|}{ Bicycle } & \multicolumn{2}{|l|}{ Car Driver } & \multicolumn{2}{|l|}{ Car Passenger } & \multicolumn{2}{|l|}{ Bus } \\
\hline & Coefficient & & Coefficient & & Coefficient & & Coefficient & \\
\hline Constant & 0.257213 & * & -3.808721 & * & 0.493503 & * & -0.021052 & * \\
\hline Age & 59.017087 & $*$ & 568.236398 & $*$ & -101.073373 & $*$ & -316.625246 & $*$ \\
\hline $\mathrm{x}$ Lat2 & 11.589239 & * & 11.188550 & * & -5.637576 & $*$ & -0.601333 & $*$ \\
\hline $\mathrm{x}$ Lat & -536.871624 & * & -594.027578 & $*$ & 214.469920 & $*$ & 22.950915 & * \\
\hline $\mathrm{x}$ Lat $\mathrm{x}$ Lon & 173.913734 & * & 145.544569 & * & -98.090232 & * & -11.099466 & $*$ \\
\hline x Lon & -8693.940767 & * & -9469.761726 & * & 3252.415166 & $*$ & 144.064206 & * \\
\hline x Lon2 & -231.371066 & * & -601.762340 & $*$ & -179.308305 & $*$ & -62.989885 & * \\
\hline x Dist CBD & 0.002477 & * & 0.006579 & * & 0.008908 & * & 0.002738 & * \\
\hline Age2 & 0.208035 & * & 3714.784113 & $*$ & -363.325975 & * & -2003.999087 & * \\
\hline $\mathrm{x}$ Lat2 & -2.347550 & * & -0.849390 & * & 0.189431 & * & -2.786547 & * \\
\hline $\mathrm{x}$ Lat & 103.064479 & * & -39.378501 & * & 4.538822 & $*$ & 168.252798 & * \\
\hline $\mathrm{x}$ Lat $\mathrm{x}$ Lon & -36.871129 & * & -42.864733 & * & 7.723402 & $*$ & -26.137624 & * \\
\hline $\mathrm{x}$ Lon & 1659.119484 & * & 2185.364039 & * & -196.909428 & $*$ & 1188.182729 & * \\
\hline x Lon2 & 14.771851 & * & 64.926867 & * & 25.122612 & $*$ & 13.036327 & * \\
\hline x Dist CBD & 0.000010 & & -0.000457 & $*$ & -0.001100 & $*$ & -0.000002 & \\
\hline Female & -9.721566 & * & 162.024196 & * & -48.293046 & $*$ & -26.375143 & $*$ \\
\hline x Lat2 & -0.198935 & * & 15.981126 & * & -6.143946 & $*$ & 1.910431 & * \\
\hline $\mathrm{x}$ Lat & 48.681273 & * & -670.854034 & * & 308.655623 & * & -91.423342 & * \\
\hline $\mathrm{x}$ Lat $\mathrm{x}$ Lon & 9.356500 & $*$ & 255.180091 & $*$ & -73.392126 & $*$ & 26.987261 & $*$ \\
\hline x Lon & 872.980191 & * & $-10,370.072817$ & $*$ & 4561.984258 & $*$ & -1498.550403 & $*$ \\
\hline x Lon2 & 237.203508 & * & 104.970553 & * & 264.653753 & * & -67.083967 & * \\
\hline x Dist CBD & 0.001757 & * & -0.002988 & * & -0.000971 & * & 0.002325 & * \\
\hline Employed & 44.802075 & $*$ & -1.800939 & $*$ & -19.824993 & $*$ & -11.172276 & * \\
\hline x Lat2 & 3.604717 & * & 7.639156 & * & 2.170157 & * & -1.426194 & * \\
\hline $\mathrm{x}$ Lat & -84.965063 & * & -142.522426 & * & -93.548643 & * & 81.958002 & $*$ \\
\hline $\mathrm{x}$ Lat $\mathrm{x}$ Lon & 78.960167 & * & 178.347825 & $*$ & 28.575083 & * & -10.861506 & * \\
\hline x Lon & -1128.403858 & * & -1760.604444 & * & -1231.441837 & $*$ & 1121.136706 & * \\
\hline $\mathrm{x}$ Lon2 & 420.621386 & * & 1092.557498 & * & 3.685472 & * & 122.044349 & * \\
\hline x Dist CBD & -0.009015 & * & -0.007930 & $*$ & -0.012930 & $*$ & -0.018221 & * \\
\hline Unemployed & -10.240727 & $*$ & -31.238073 & * & -2.442526 & $*$ & -9.653250 & * \\
\hline x Lat2 & 4.343593 & $*$ & 7.149145 & $*$ & 0.556634 & $*$ & -1.714387 & * \\
\hline $\mathrm{x}$ Lat & -196.678708 & * & -154.759240 & $*$ & -59.847677 & * & 72.125433 & * \\
\hline $\mathrm{x}$ Lat $\mathrm{x}$ Lon & 68.523897 & * & 161.970249 & * & -8.509376 & $*$ & -20.171492 & * \\
\hline x Lon & -3298.182441 & $*$ & -2142.103964 & $*$ & -763.940541 & $*$ & 807.192994 & $*$ \\
\hline $\mathrm{x}$ Lon2 & -70.725905 & $*$ & 890.574693 & $*$ & -205.865309 & $*$ & -11.949255 & $*$ \\
\hline x Dist CBD & 0.001372 & * & -0.003578 & $*$ & -0.008548 & * & -0.011878 & * \\
\hline Homemaker & -11.624779 & $*$ & 18.549815 & $*$ & 21.836178 & $*$ & -5.983425 & $*$ \\
\hline $\mathrm{x}$ Lat2 & 4.167724 & $*$ & 2.522755 & $*$ & 3.414515 & $*$ & -4.523395 & $*$ \\
\hline $\mathrm{x}$ Lat & 22.095877 & $*$ & 28.906657 & $*$ & -173.227404 & $*$ & 48.581866 & $*$ \\
\hline $\mathrm{x}$ Lat $\mathrm{x}$ Lon & 132.602514 & * & 78.815932 & * & 33.579698 & * & -121.891147 & * \\
\hline x Lon & 688.037444 & $*$ & 1003.523820 & $*$ & -2225.112856 & $*$ & 526.762824 & $*$ \\
\hline $\mathrm{x}$ Lon2 & 1181.199706 & $*$ & 814.844567 & $*$ & -135.570616 & $*$ & -884.967581 & $*$ \\
\hline x Dist CBD & -0.012048 & * & -0.009279 & * & -0.012042 & * & -0.006867 & * \\
\hline Retired & -15.628043 & * & 98.313740 & * & -14.320662 & * & -27.890992 & $*$ \\
\hline x Lat2 & 1.082815 & * & 5.051318 & * & -4.240966 & $*$ & 1.728547 & $*$ \\
\hline $\mathrm{x}$ Lat & 78.584230 & * & -30.838246 & $*$ & 127.141993 & $*$ & -78.735567 & * \\
\hline $\mathrm{x}$ Lat $\mathrm{x}$ Lon & 51.274891 & * & 132.881761 & * & -105.473602 & * & 19.579988 & * \\
\hline x Lon & 1778.060953 & * & 287.852856 & $*$ & 2809.470332 & $*$ & -1036.428256 & * \\
\hline x Lon2 & 739.820476 & * & 1111.281895 & * & -310.321838 & $*$ & -41.751588 & * \\
\hline x Dist CBD & -0.015446 & * & -0.016307 & $*$ & -0.002348 & $*$ & -0.021710 & * \\
\hline Land Use Type: Industrial & 0.970141 & * & 1.649628 & * & 1.807279 & * & 0.707925 & * \\
\hline Land Use Type: Medium & 0.214288 & * & 0.295141 & $*$ & 0.432478 & $*$ & 0.135478 & $*$ \\
\hline Land Use Type: New Urban & 0.000968 & * & 0.863843 & $*$ & 1.264870 & $*$ & 1.813088 & * \\
\hline Land Use Type: Residential & 0.534994 & * & 0.610086 & $*$ & 0.484239 & $*$ & 0.646945 & * \\
\hline Land Use Type: Rural & 0.172042 & $*$ & 0.880376 & $*$ & 1.569607 & $*$ & 0.526331 & * \\
\hline
\end{tabular}

Note: Walking is the reference mode.

$$
{ }^{*} p<.05 . p<.10 \text {. }
$$

Residential fabrics seem to be somewhat more supportive of active travel, as seen from their relatively smaller coefficients for travel by auto.

Next, we map the variations in the propensity to travel according to attributes that were spatially expanded. This is the most effective way of understanding how these attributes influence travel by the various modes. To do this, we create the profiles shown in Table 4. Based on these profiles, we can use the model to estimate the probabilities of traveling by different modes. The results of this exercise appear in Fig. 4. The labels in the figure indicate the age, gender, and mode of travel. Every profile corresponds to employed people, we have not
Table 4

Profile for visualization of results.

\begin{tabular}{llll}
\hline Profile & Gender & Age & Occupation \\
\hline Profile 1 & Female & 20 & Employed \\
Profile 2 & Male & 20 & Employed \\
Profile 3 & Female & 60 & Employed \\
Profile 4 & Male & 60 & Employed \\
\hline
\end{tabular}




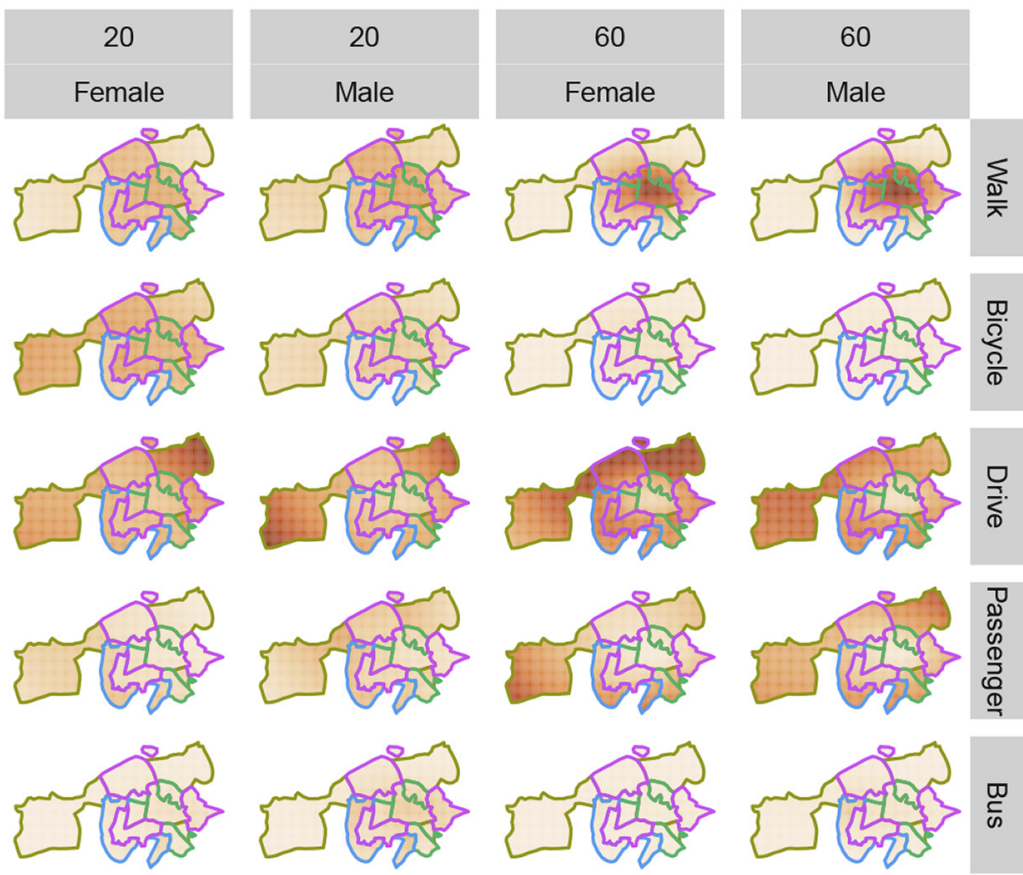

\section{Fabric

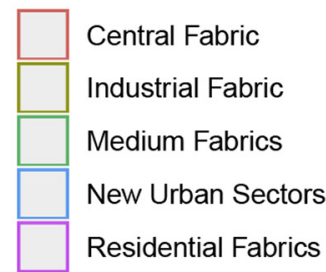

Probability

0.8

0.6

0.4

0.2

Fig. 4. Probability of traveling by different modes by age, gender, and geograpical origin of trip.

distinguished levels of occupation in order to present a compact discussion of the results.

The figure contains the four profiles defined in the table (columns) and represents the probability of mode use, for each mode considered in this study (rows). Recall that the spatial expansion is based on the origin of the trip, hence the probabilities in the figure correspond to the probability of using a mode for a trip that begins at that location.

It can be seen in the figure that the probability of walking is generally high in the Central and Medium Fabrics near the CBD of VitoriaGasteiz. This probability tends to decline with distance from the CBD, and when transitioning to Residential Fabrics and New Urban Sectors. With respect to age, we notice that the spatial variation of the probability of walking tends to be flatter when we examine the two younger profiles (i.e., 20 years old). When we inspect the two senior profiles (i.e., 60 years old), in contrast, the probability of walking appears to be substantially higher, however, it also drops more rapidly outside of the Central and Medium Fabrics near the CBD.

With respect to cycling, we see that although this mode is not as probable as walking, it is still more probable in the two young profiles compared to Profiles 3 and 4. Among younger travelers, cycling remains relatively popular among females, whereas for the two 60 -yearold profiles there is evidence of only very small gender differences. The probability of cycling by young women is moderately high in the Central and Medium Fabrics, and also in the industrial region located in the west of the city. The spatial variations, however, tend to be flatter, perhaps because the probability of cycling tends to be relative lower across the region.

Regarding travel as a driver of a car, the highest probabilities are located outside of the Central Fabric. In the case of the young profiles, the probability of driving is very high in the Industrial Fabrics, while in the case of the 60-year-old profiles the probability of car use is very high in every fabric outside of the Central Fabric. The probability of using a car as passenger is high in the case of profiles 3 and 4 and more marked in the more peripheral parts of the city, while in profiles 1 and 2 this probability remains low. The probability for bus use is low for every profile and location, and only young men (profile 2 ) show slightly higher probabilities for using public transport in New Urban sectors and Residential fabrics located in the south, west and east of the city.

A more refined perspective of the differences in the use of active modes of travel can be obtained from examining the odd-ratios of the probabilities. Figs. 5 to 8 help to explain the particularities of active mode use in Vitoria-Gasteiz for different demographic and spatial profiles.

Fig. 5 plots the odd-ratios of the probabilities for walking of Profile 2 with respect to Profile 1 (young male and young female). According to the results, young men are more likely than women to walk in the Central and Medium Fabrics in the center of the city, and also in the Residential Fabric but only in the east of the city center. However, for trips that begin further away from the center, this trend is inverted, and women are more likely to walk than men - although it is important to note that in those regions the probability of walking is already relatively low. Active travel by bicycle in young profiles shows a strikingly different spatial trend. Fig. 6 plots the odd-ratios of bicycle, again looking at Profiles 2 with respect to Profile 1 (young men and young female). The results indicate that bicycle use by women is substantially higher across the region. Locations closest to the CBD and the periphery of the city (where Industrial and New Urban Development are common) show a particularly high prevalence of bicycle use by women, which is less pronounced in the belt that surrounds the Central Fabric, with the presence of Medium and Residential Fabrics.

The comparison of the probability of use of active modes to driving also leads to interesting spatial variations in Vitoria-Gasteiz. For example, the ratio of odds for cycling and driving in young females (Fig. 7) shows that in the Central Fabric, and in the Residential and Medium Fabrics that surround it to the south, west and northwest, women are up to 1.6 times more likely to cycle than to drive. However, in the Industrial Fabrics, the New Urban Developments in the south and west, and in the Residential Fabric in the east, the use of car prevails. Walking is always highly more probable than driving for young men in the whole city, as it is presented in Fig. 7, where the ratio of odds for walking to driving is plotted using Profile 2 (young male).

\section{Conclusions}

The objective of this paper has been to investigate the factors that influence the propensity to travel by active modes of transportation in Vitoria-Gasteiz in Spain. The analysis was based on the application of multinomial logistic regression. The availability of georeferenced 


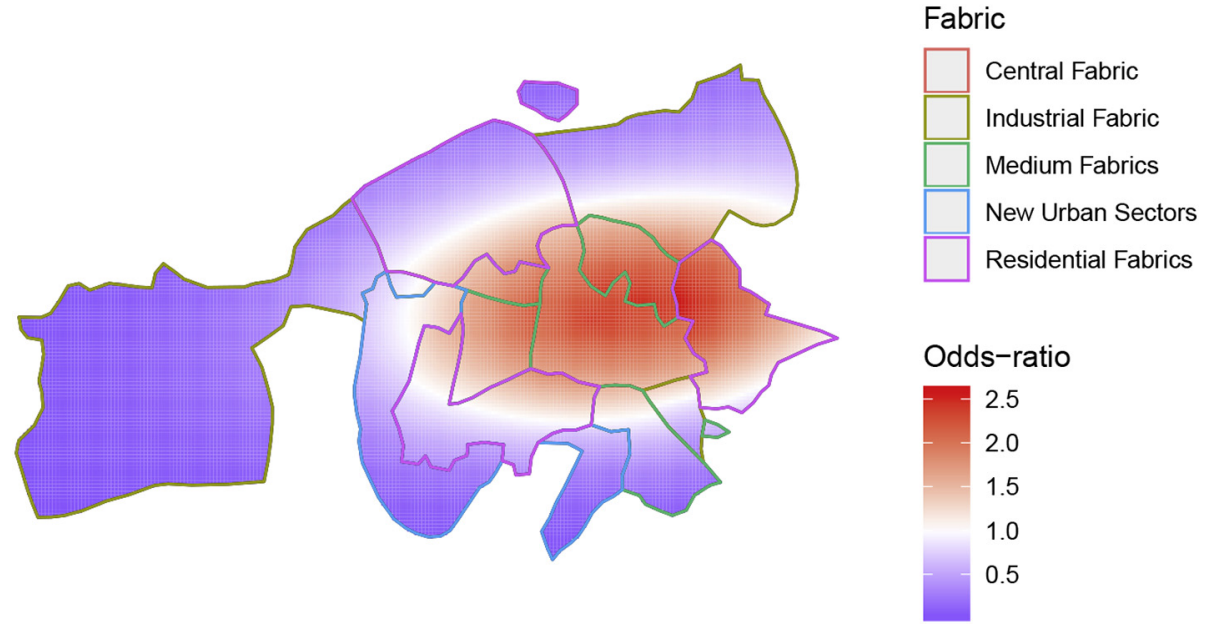

Fig. 5. Odds-ratio of the probability of walking, Profile 2 (Young Male) to Profile 1 (Young Female).

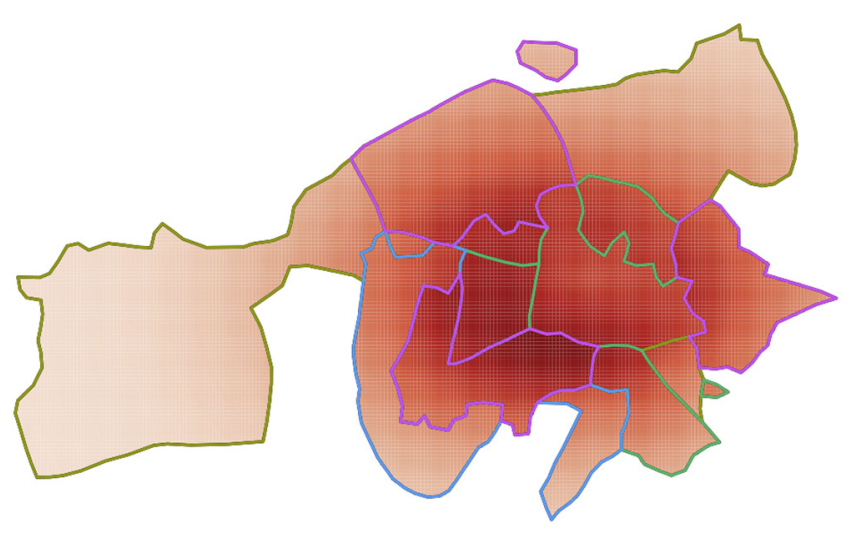

Fabric

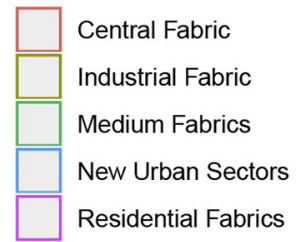

Odds-ratio

-0.25
-0.20
-0.15
0.10
-0.05

Fig. 6. Odds-ratio of the probability to cycle Profile 2 (Young Male) to Profile 1 (Young Female).

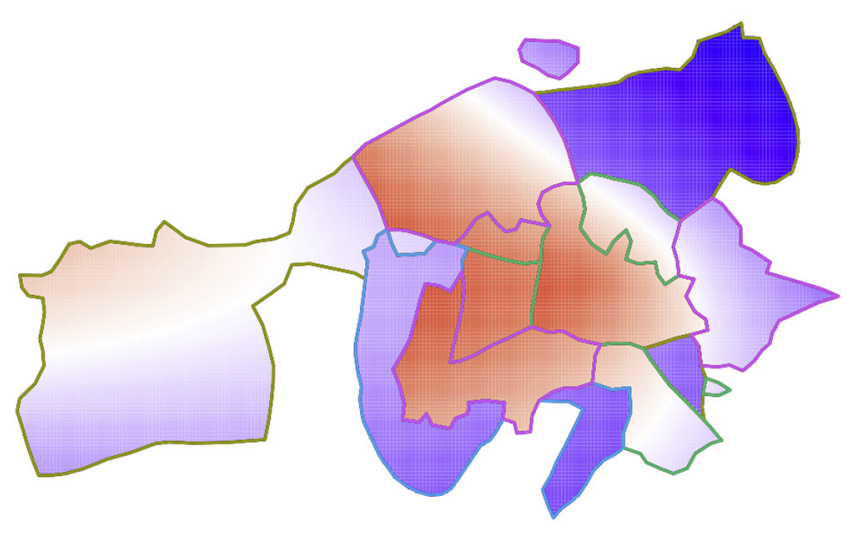

Odds-ratio

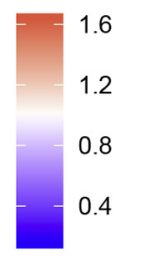

\section{Fabric}

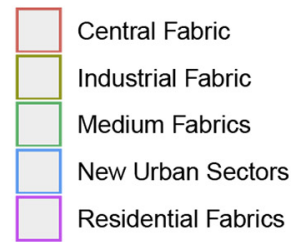

Fig. 7. Odds-ratio of the probability of cycling to driving, Profile 1 (Young Female).

information also allowed us to develop a model with spatially-varying coefficients. The results provide evidence that travel behavior - expressed as the probability of using different modes - has an important geographical component.

The focus of the analysis was on the socio-economic and demographic factors that influence active travel. The study of different socio- demographic and geographic profiles helps to identify the areas and segments of population that are more active in their mobility, distinguishing among different types of urban fabrics.

The study of the spatial variation of mode use provides important information about the locations where interventions may be required to encourage active travel. Walking, despite being quite popular overall 


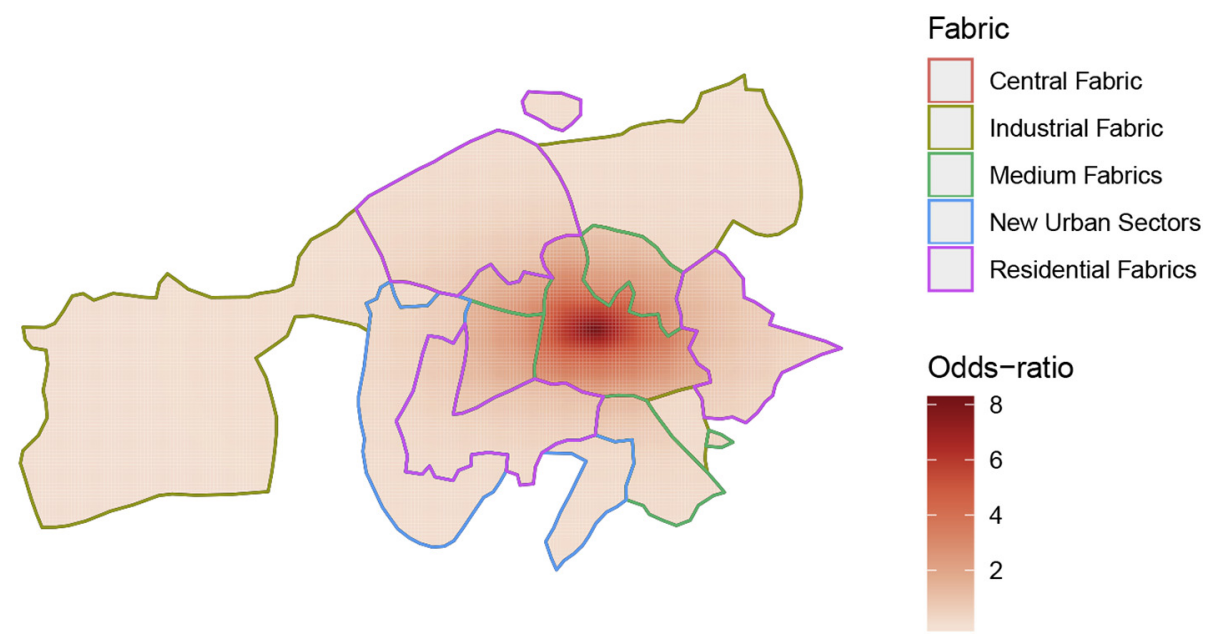

Fig. 8. Odds-ratio of the probability of walking to driving, Profile 2 (Young male).

(see Table 2), also tends to be fairly concentrated spatially, in particular in the Central Fabric, while its popularity declines markedly in the Industrial, Rural, and New Urban fabrics. The level of active travel among women is notable, since they are more likely to walk than men in many cases, and more likely to cycle than men. Bicycle is the third most important transport mode in the city and preferred at younger ages, while walking becomes the most probable mode for seniors.

The active travel behavior in Vitoria -Gasteiz across the age span is coincident with previous studies. For example, seniors are more likely to walk than the younger profiles, as it was also reported by other authors (Bocker et al., 2017). However, our analysis allows to modulate the results attending to urban configuration characteristics that in Vitoria-Gasteiz the difference is substantially higher in the Central Fabric, which is characterized by a high population density and mixed land uses. Our results about cycling by gender, in contrast, are different from the general trend reported reported by other authors, who often find that women are less likely to cycle than men (Beecham and Wood, 2014; Grudgings et al., 2018; Mitra and Nash, 2018). This result may be an indication of a turnaround in women mobility (Tilley and Houston, 2016).

The findings reported in this paper offer valuable insights regarding urban fabrics at the origin of trips, and how these fabrics correlate with the use of active modes. For example, active travel tends to be more common in the Central, Residential, and Medium Type fabrics. In contrast, urban fabrics classified as New Urban Developments appear to be less supportive of active modes, and car use there is in fact tends to be quite similar to Rural development, other things being equal. This seems to be a combination of lower density developments, and greater heterogeneity in type of development. The identification of these areas is of particular interest, given that these zones are where initiatives could be intensified to modify this trend. Further research may be focused into qualitative research and more in-detail urban design issues that may be affecting active mobility in these new urban developments.

In summary, the results indicate that more compact and mixed uses of land tend to be more favorable to the use of active modes, a relevant result in Vitoria-Gasteiz, where car trips that could be replaced by active modes could reach $40 \%$, according to (Delso et al., 2018). More generally, the findings in this paper support previous research on the factors that associate with active travel (e.g., Cervero, 1996; Ewing and Cervero, 2010; Naess, 2012), and offer validation of previous research but for smaller urban areas (Wolday, 2018) and other findings from Nordic Countries (Naess and Jensen, 2004; Wolday, 2018).

This study has limitations that open up other new opportunities for further research. We have not discussed the implications of the socioeconomic aspects and their relationship with the active travel. Occupation was found to be a significant variable in the multinomial logistic regression, but its levels were not included in the profiles constructed to identify, explain and plot the areas and segments of population that are more active in their mobility. Further work will explore additional socioeconomic status measures and more disaggregated indicators of land uses and the built environment.

\section{Acknowledgments}

This research was initiated while the first author was visiting at McMaster Institute for Transportation and Logistics. The authors are grateful by the hospitality of the Institute during Dr. Martn's visit. This paper was produced under the framework of the following projects: "Desarrollo de Aplicaciones SIG para la Implementación de Indicadores de Fragmentación Urbana y Mejora de la Movilidad" funded by the Universidad Politécnica de Madrid, research project no. RP151320028, and "DESPACIO" (TRA2017-88058-R) funded by the Spanish Ministry of Science in the "Programa Estatal de I + D + i Orientada a los Retos de la Sociedad". The authors wish to acknowledge the financial support of the "Programa Propio de I+D +i 2018 Universidad Politécnica de Madrid" for the grant awarded to Belén Martín in the call "Convocatoria de ayudas a PDI e Investigadores Doctores para realizar Estancias de Investigación Internacional igual o superior a un mes". The authors would also like to thank the Centro de Estudios Ambientales (CEA Victoria-Gasteiz) and the "TRANSBICI" Project (TRA2010-17035) for supplying the HTS. The following R packages were used in the course of this investigation and the authors wish to acknowledge their developers: knitr (Xie, 2015; Xie, 2018), tidyverse (Wickham, 2017), dplyr (Wickham et al., 2018), rgdal (Bivand et al., 2018), tmap and tmaptools (Tennekes, 2018), rworldmap (South, 2011), kableExtra (H. Zhu, 2018), png (Urbanek, 2013), gridExtra (Auguie, 2017), gridGraphics (Murrell and Wen, 2018), rgeos (Bivand and Rundel, 2018), gtable (Wickham, 2016), plyr (Wickham, 2011), nnet (Venables and Ripley, 2002), and reshape2 (Wickham, 2007).

\section{References}

Agencia de Ecología Urbana de Barcelona, 2010. Plan de Indicadores de Sostenibilidad Urbana de Vitoria-Gasteiz. Report 2009. http://www.vitoria-gasteiz.org/wb021/ http/contenidosEstaticos/adjuntos/es/89/14/38914.pdf.

Auguie, Baptiste, 2017. GridExtra: Miscellaneous Functions for "Grid” Graphics. https:// CRAN.R-project.org/package $=$ gridExtra.

Ayuntamiento de Vitoria-Gasteiz, 2011. Información práctica sobre El área comercial Y de negocios del centro urbano de La capital de Euskadi. Report. https://www.vitoriagasteiz.org/docs/wb021/contenidosEstaticos/adjuntos/es/39/78/43978.pdf (36 pages).

Ayunramiento de Vitoria-Gasteiz, 2016. Informe de Población Vitoria-Gasteiz 2016. Movimientos Demográficos 2015. Padrón Municipal de Habitantes. Report. https:// www.vitoria-gasteiz.org/docs/wb021/contenidosEstaticos/adjuntos/es/40/39/ 64039.pdf (25 pages). 
Beecham, Roger, Wood, Jo, 2014. Exploring gendered cycling behaviours within a largescale behavioural data-set. Transp. Plan. Technol. 37 (1), 83-97 Taylor \& Francis.

Best, Henning, Lanzendorf, Martin, 2005. Division of labour and gender differences in metropolitan car use: an empirical study in cologne, Germany. J. Transp. Geogr. 13 (2), 109-121 Elsevier.

Bivand, Roger, Rundel, Colin, 2018. Rgeos: Interface to Geometry Engine - Open Source ('Geos'). https://CRAN.R-project.org/package = rgeos.

Bivand, Roger, Keitt, Tim, Rowlingson, Barry, 2018. Rgdal: Bindings for the 'Geospatial' Data Abstraction Library. https://CRAN.R-project.org/package = rgdal.

Bocker, Lars, van Amen, Patrick, Helbich, Marco, 2017. Elderly travel frequencies and transport mode choices in greater rotterdam, the Netherlands. Transportation 44 (4), 831-852 Springer.

Casetti, Emilio, 1972. Generating Models by the Expansion Method: Applications to Geographical Research. Geogr. Anal. 4 (1), 81-91 Wiley Online Library.

Cervero, Robert, 1996. Mixed land-uses and commuting: evidence from the american housing survey. Transport. Res. Part A 30 (5), 361-377 Elsevier.

Christiansen, Lars B., Cerin, Ester, Badland, Hannah, Kerr, Jacqueline, Davey, Rachel, Troelsen, Jens, Van Dyck, Delfien, et al., 2016. International comparisons of the associations between objective measures of the built environment and transport-related walking and cycling: ipen adult study. J. Transp. Health 3 (4), 467-478 Elsevier.

Delso, Javier, Martin, Belen, Ortega, Emilio, 2018. Potentially replaceable car trips: assessment of potential modal change towards active transport modes in vitoria-gasteiz Sustainability 10 (10), 3510 Multidisciplinary Digital Publishing Institute.

ESRI, 2015. Computer Program. https://support.esri.com/es/Products/Desktop/arcgisdesktop/arcmap/10-3-1\#overview.

Ewing, Reid, Cervero, Robert, 2010. Travel and the built environment: a meta-analysis. J. Am. Plan. Assoc. 76 (3), 265-294 Taylor \& Francis.

Gatrell, Anthony C., 2013. Therapeutic mobilities: walking and 'steps' to wellbeing and health. Health Place 22, 98-106 Elsevier.

GitHub Contributors, 2018. Computer Program. https://github.com/googlemaps/googlemaps-services-python.

Goodman, Anna, 2013. Walking, cycling and driving to work in the english and welsh 2011 census: trends, socio-economic patterning and relevance to travel behaviour in general. PLoS ONE 8 (8), e71790 Public Library of Science.

Grannis, Rick, 2009. From the Ground up: Translating Geography into Community through Neighbour Networks. Princeton University Press.

Grudgings, Nick, Hagen-Zanker, Alex, Hughes, Susan, Gatersleben, Birgitta, Woodall, Marc, Bryans, Will, 2018. Why don't more women cycle? an analysis of female and male commuter cycling mode-share in england and wales. J. Transp. Health 10, 272-283 Elsevier.

Hartog, De, Johan, Jeroen, Boogaard, Hanna, Nijland, Hans, Hoek, Gerard, 2010. Do the health benefits of cycling outweigh the risks? Environ. Health Perspect. 118 (8), 1109 National Institute of Environmental Health Science.

International Transport Forum, 2012. Pedestrian Safety, Urban Space and Health. https:// doi.org/10.1787/9789282103654-en.

Kerr, Jacqueline, Sallis, James F., Owen, Neville, De Bourdeaudhuij, Ilse, Cerin, Ester, Sugiyama, Takemi, Reis, Rodrigo, et al., 2013. Advancing science and policy through a coordinated international study of physical activity and built environments: IPEN adult methods. J. Phys. Act. Health 10 (4), 581-601.

Kim, Hyungkyoo, Macdonald, Elizabeth, 2016. Does wind discourage sustainable transportation mode choice? findings from San Francisco, California, USA. Sustainability 8 (3), 257 Multidisciplinary Digital Publishing Institute.

Kim, Sungyop, Ulfarsson, Gudmundur, 2004. Travel mode choice of the elderly: effects of personal, household, neighborhood, and trip characteristics. Transp. Res. Rec. 1894, 117-126 Transportation Research Board of the National Academies.

Litman, Todd, 2003. Measuring Transportation: Traffic, Mobility and Accessibility. Inst Transport. Eng. ITE J. 73 (10) (Institute of Transportation Engineers: 28).

Lois, David, Moriano, Juan Antonio, Rondinella, Gianni, 2015. Cycle commuting intention: a model based on theory of planned behaviour and social identity. Transport. Res. Part F 32, 101-113 Elsevier.

Mackenbach, Joreintje Dingena, Randal, Edward, Zhao, Pengjun, Howden-Chapman, Philippa, 2016. The Influence of Urban Land-Use and Public transport facilities on Active Commuting in Wellington, New Zealand: active transport forecasting using the Wilute Model. Sustainability 8 (3), 242 Multidisciplinary Digital Publishing Institute.

Mercado, Ruben, Paez, Antonio, 2009. Determinants of distance traveled with a focus on the elderly: a multilevel analysis in the hamilton cma, canada. J. Transp. Geogr. 17 (1), 65-76 Elsevier.

Mitra, Raktim, Nash, Sean, 2018. Can the built environment explain gender gap in cycling? an exploration of university students' travel behavior in Toronto, Canada. Int. J. Sustain. Trans. 1-10 Taylor \& Francis.

Moniruzzaman, M., Paez, A., Habib, K.M.N., Morency, C., 2013. Mode use and trip length of seniors in Montreal. J. Art J. Trans. Geogr. 30, 89-99. https://doi.org/10.1016/j. jtrangeo.2013.03.00710.1016/j.jtrangeo.2013.03.007.

Moniruzzaman, Md, Paez, Antonio, Morency, Catherine, 2014. Compliance potential mapping: a tool to assess potential contributions of walking towards physical activity guidelines. BMC Public Health 14 (1), 511 BioMed Central.

Morency, Catherine, Demers, Marie, Lapierre, Lucie, 2007. How Many Steps Do You Have in Reserve?: Thoughts and Measures About a Healthier Way to Travel. Transp. Res. Rec. 2002, 1-6 Transportation Research Board of the National Academies.

Morency, Catherine, Paez, Antonio, Roorda, Matthew J., Mercado, Ruben, Farber, Steven, 2011. distance traveled in three canadian cities: spatial analysis from the perspective of vulnerable population segments. J. Transp. Geogr. 19 (1), 39-50 Elsevier.

Morency, Catherine, Demers, Marie, Poliquin, Eric, 2014. shifting short motorized trips to walking: the potential of active transportation for physical activity in montreal. J. Transp. Health 1 (2), 100-107 Elsevier.

Muñoz, B., 2016. Integrating Bicycle Option in Mode Choice Models Through Latent
Variables. Ph.D. Thesis. Universidad Politécnica de Madrid, Spain.

Muñoz, B., Rondinella, G., 2017. Informe de Evaluación Del Plan de Movilidad Sostenible Y Espacio Público Y Del Plan Director de Movilidad Ciclista de Vitoria-Gasteiz. Vitoria-Gasteiz: Centro de Estudios Ambientales. Report. Centro de Estudios Ambientales. http://www.vitoria-gasteiz.org/wb021/http/contenidosEstaticos/ especial/2017/0406/PMSEP_evaluacion_2017.pdf.

Murrell, Paul, Wen, Zhijian, 2018. GridGraphics: Redraw Base Graphics Using 'Grid' Graphics. https://CRAN.R-project.org/package= gridGraphics.

Naess, Petter, 2012. Urban Form and Travel Behavior: Experience from a Nordic Context. J. Trans. Land Use 5 (2), 21-45 JSTOR.

Naess, Petter, Jensen, Ole B., 2004. urban structure matters, even in a small town. J. Environ. Plan. Manag. 47 (1), 35-57 Taylor \& Francis.

Naess, Petter, Strand, Arvid, Wolday, Fitwi, Stefansdottir, Harpa, 2017. Residential Location, Commuting and Non-Work Travel in Two Urban Areas of Different Size and with Different Center Structures. Progress in Planning. Elsevier.

Paez, Antonio, Scott, Darren, Potoglou, Dimitris, Kanaroglou, Pavlos, Newbold, K. Bruce, 2007. Elderly Mobility: Demographic and Spatial Analysis of Trip Making in the Hamilton Cma, Canada. Urban Stud. 44 (1), 123-146 Sage Publications Sage UK: London, England.

Potoglou, Dimitris, Arslangulova, Botakoz, 2017. Factors Influencing Active Travel to Primary and Secondary Schools in Wales. Transp. Plan. Technol. 40 (1), 80-99 Taylor \& Francis.

Pucher, John, Buehler, Ralph, 2008. making cycling irresistible: lessons from the netherlands, denmark and germany. Transp. Rev. 28 (4), 495-528 Taylor; Francis.

Rondinella, G., 2015. Considering Cycling for Commuting: The Role of Mode Familiarity. Ph.D. Thesis. Universidad Politécnica de Madrid, Spain.

Roorda, M.J., Paez, A., Morency, C., Mercado, R., Farber, S., 2010. trip generation of vulnerable populations in three canadian cities: a spatial ordered probit approach. J. Art. Transport. 37 (3), 525-548. https://doi.org/10.1007/s11116-010-9263-310. 1007/s11116-010-9263-3.

Saelens, Brian E., Sallis, James F., Black, Jennifer B., Chen, Diana, 2003. neighborhoodbased differences in physical activity: an environment scale evaluation. Am. J. Public Health 93 (9), 1552-1558 American Public Health Association.

Schmocker, Jan-Dirk, Quddus, Mohammed A., Noland, Robert B., Bell, Michael G.H., 2008. mode choice of older and disabled people: a case study of shopping trips in London. J. Transp. Geogr. 16 (4), 257-267 Elsevier.

Schwanen, Tim, Dieleman, Frans M., Dijst, Martin, 2001. Travel Behaviour in Dutch Monocentric and Policentric Urban Systems. J. Transp. Geogr. 9 (3), 173-186 Elsevier.

Simons, Dorien, De Bourdeaudhuij, Ilse, Clarys, Peter, de Geus, Bas, Vandelanotte, Corneel, Cauwenberg, Jelle Van, Deforche, Benedicte, 2017. Choice of Transport Mode in Emerging Adulthood: Differences Between Secondary School Students, Studying Young Adults and Working Young Adults and Relations with Gender, Ses and Living Environment. Transport. Res. Part A 103, 172-184 Elsevier.

South, Andy, 2011. Rworldmap: a new R package for mapping global data. R J. 3 (1) 35-43. http://journal.r-project.org/archive/2011-1/RJournal_2011-1_South.pdf.

Steinmetz-Wood, Madeleine, Kestens, Yan, 2015. Does the effect of walkable built environments vary by neighborhood socioeconomic status. Prev. Med. 81, 262-267 Elsevier.

Stevens, Mark R., 2017. Does compact development make people drive less? J. Am. Plan. Assoc. 83 (1), 7-18 Taylor Francis.

Tennekes, Martijn, 2018. Tmap: thematic maps in R. J. Stat. Softw. 84 (6), 1-39. https:// doi.org/10.18637/jss.v084.i0610.18637/jss.v084.i06.

Tilley, Sara, Houston, Donald, 2016. The gender turnaround: young women now travelling more than young men. J. Transp. Geogr. 54, 349-358 Elsevier.

United Nations, 2014. World urbanization prospects: the 2014 revision, highlights. In: Department of Economic and Social Affairs. United Nations, Population Division.

Urbanek, Simon, 2013. Png: Read and Write Png Images. https://CRAN.R-project.org/ package $=$ png

US Department of Health and Human Services and others, 2008. 2008 Physical Activity Guidelines for Americans: Be Active, Healthy, and Happy!. Http://Www (Health. Gov/Paguidelines).

Venables, W.N., Ripley, B.D., 2002. Modern Applied Statistics with S. Springer, Fourth. New York. http://www.stats.ox.ac.uk/pub/MASS4.

Whalen, K.E., Paez, A., Bhat, C., Moniruzzaman, M., Paleti, R., 2012. T-communities and sense of community in a university town: evidence from a student sample using a spatial ordered-response model. J. Art. Urban Stud. 49 (6), 1357-1376. https://doi. org/10.1177/004209801141194210.1177/0042098011411942.

Wickham, Hadley, 2007. Reshaping data with the reshape package. J. Stat. Softw. 21 (12), 1-20. http://www.jstatsoft.org/v21/i12/.

Wickham, Hadley, 2011. The Split-apply-combine strategy for data analysis. J. Stat. Softw. 40 (1), 1-29. http://www.jstatsoft.org/v40/i01/.

Wickham, Hadley, 2016. Gtable: Arrange 'Grobs' in Tables. https://CRAN.R-project.org/ package $=$ gtable.

Wickham, Hadley, 2017. Tidyverse: Easily Install and Load the 'Tidyverse'. https://CRAN. R-project.org/package $=$ tidyverse.

Wickham, Hadley, François, Romain, Henry, Lionel, Müller, Kirill, 2018. Dplyr: A Grammar of Data Manipulation. https://CRAN.R-project.org/package=dplyr.

Wolday, Fitwi, 2018. Built environment and Car driving distance in a Small City context. J. Trans. 11 (1), 747-767 Land Use.

World Health Organization, 2017. Physical activity, www document. Report. http:// www.who.int/mediacentre/factsheets/fs385/en/.

Xie, Yihui, 2015. Dynamic Documents with R and Knitr, 2nd ed. Boca Raton, Florida, Chapman; hall/CRC. https://yihui.name/knitr/.

Xie, Yihui, 2018. Knitr: a General-Purpose Package for Dynamic Report Generation in R. https://yihui.name/knitr/. 
Zhu, Hao, 2018. KableExtra: construct Complex Table with 'Kable' and Pipe Syntax. https://CRAN.R-project.org/package = kableExtra.

Zhu, Xuemei, Yu, Chia-Yuan, Lee, Chanam, Lu, Zhipeng, Mann, George, 2014. A

Retrospective study on changes in residents' physical activities, social interactions, and neighborhood cohesion after moving to a walkable community. Prev. Med. 69, S93-S97 Elsevier. 